\title{
Ordered Weighted Averaging Operators and their Generalizations with Applications in Decision Making
}

\author{
F. Kouchakinejad ${ }^{1}$ and A. Šipošová2,**
}

\begin{abstract}
The definition of ordered weighted averaging (OWA) operators and their applications in decision making are reviewed. Also, some generalizations of OWA operators are studied and then, the notion of 2-symmetric OWA operators is introduced. These generalizations are illustrated by some examples.
\end{abstract}

Keywords: Aggregation function, Fuzzy measure, Generalizations of OWA operators, 2symmetric OWA operators.

Manuscript was received on 11/08/2017, revised on 07/11/2017 and accepted for publication on 19/11/2017.

\section{Introduction and Preliminaries}

Aggregation functions are indispensable in real-world applications where quantitative evaluation data are required to be fused into a single numerical entry. Examples abound and include decision making with the help of aggregating scores or preferences with respect to certain alternatives, or compressing information by merging multiple origin inputs to simplify recognition and classification, and so on, all with applications in artificial intelligence, risk management, decision making, statistical inference and many other areas.

Literature on aggregation functions is abundant and we just refer to [2,9] for basic concepts and different types of such functions that have been considered. For the purpose of this contribution, an aggregation function is any mapping $A:[0,1]^{n} \rightarrow[0,1]$ which is increasing in every coordinate with $\mathrm{A}(\mathbf{0})=0$ and $\mathrm{A}(\mathbf{1})=1$.

The ordered weighted averaging (OWA) operator have been introduced by Yager [21] as a tool to deal with the problem of aggregating multi criteria objectives to form an overall decision function. This type of an aggregation function provides a parameterized family of aggregation functions which has been studied in many works. The class of OWA operators includes minimum, maximum, arithmetic mean, median and so on [6]. For an overview of results related to OWA operators see [4]. The applications of OWA operators can be seen in many areas. The most important area is decision making. So far, several works such as $[7,11,22]$ have been devoted to applications of OWA operators in the decision making and programming problems.

There are several works in the literature in which OWA operators are used as superior aggregation functions in the decision problems. This choice is mainly based on theoretical arguments. The OWA

\footnotetext{
* Corresponding Author.

${ }^{1}$ Department of Statistics, Faculty of Mathematics and Computer Science, Shahid Bahonar University of Kerman, Kerman, Iran, Email: kouchakinezhad@gmail.com.

2 Department of Mathematics and Descriptive Geometry, Faculty of Civil Engineering, Slovak University of Technology in Bratislava, Bratislava, Slovakia, Email: alexandra.siposova@stuba.sk.
} 
operator shares with other aggregation functions the properties of monotonicity and boundedness. From these properties, the property of idempotency can be deduced. If an alternative has the same evaluation in all attributes, this same value is also the overall evaluation of the alternative. The OWA operator also has the property of symmetry; rearranging values across attributes does not change the overall evaluation. This property known as is the main advantage of the OWA operator in literature.

Multi attribute decision making (MADM) methods are either compensatory or non-compensatory by nature, and thus allow (or do not allow) weak performance in one attribute to be compensated by good performance in other attributes. Using OWA operators, the possibility of compensation can be controlled due to the structure of weights by using the concept of orness, that provides additional flexibility for decision makers which is not possible in other models. Therefore, since the introduction of the OWA operator to MADM problems by Yager [21], OWA operators became the new model for aggregating multiple input arguments, presenting a unifying and generalizing formulation for uncertain decision making problems [1] and acting as the common method for aggregating information in multi attribute decision problems [16].

As said before, OWA operators can flexibly be used to represent compensatory as well as noncompensatory preferences, and it can model different degrees of compensation among attributes. Formally, this property is represented by the degree of orness of an OWA operator, which was already defined by Yager [21]. The orness of an OWA operator (i.e., of a weight vector $\mathbf{w}=\left(w_{1}, w_{2}, \ldots, w_{n}\right)$ of length $n$ ) is

$$
\operatorname{orness}(\mathbf{w})=\frac{1}{n-1} \sum_{i=1}^{n}(n-i) w_{i} .
$$

The weight vector $\mathbf{w}=(1,0, \ldots, 0)$, which focuses only on the best attribute represents the strongest possibility for compensation and has a degree of orness of one. Conversely, the weight vector $\mathbf{w}=(0,0, \ldots, 1)$ represents a purely non-compensatory model and has an orness of zero. Intermediate levels of orness correspond to situations in which some compensation of weak performance in one attribute by better performance in other attributes is possible.

Considering the importance of this field, studying OWA operators seems appears to be necessary. In this regard, in this paper, we recall the basic facts about OWA operators and review some of its generalizations with the view of applications.

OWA operator is defined by

$$
\operatorname{OWA}_{\mathbf{w}}\left(x_{1}, \ldots, x_{n}\right)=\sum_{i=1}^{n} w_{i} x_{\sigma(i)},
$$

where $\sigma:\{1, \ldots, n\} \rightarrow\{1, \ldots, n\}$ is a permutation satisfying $x_{\sigma(1)} \geq \cdots \geq x_{\sigma(n)}$, and $\mathbf{w}=$ $\left(w_{1}, \ldots, w_{n}\right) \in[0,1]^{n}$, with $\sum_{i=1}^{n} w_{i}=1$.

Observe that OWA operators can be characterized also axiomatically as symmetric comonotone additive aggregation functions on $[0,1]$; see $[8,18]$. This means monotone functions $A:[0,1]^{n} \rightarrow$ $[0,1]$ satisfying the boundary conditions $A(\mathbf{0})=0, A(\mathbf{1})=1$, which are symmetric, i.e., $A\left(x_{1}, \ldots, x_{n}\right)=A\left(x_{\sigma(1)}, \ldots, x_{\sigma(n)}\right)$, for any permutation $\sigma:\{1, \ldots, n\} \rightarrow\{1, \ldots, n\}$ and any $\mathbf{x} \in$ 
$[0,1]^{n}$, and comonotone additive, i.e., $A(\mathbf{x}+\mathbf{y})=A(\mathbf{x})+A(\mathbf{y})$ for any $\mathbf{x}, \mathbf{y}, \mathbf{x}+\mathbf{y} \in[0,1]^{n}$ such that $\left(x_{i}-x_{j}\right)\left(y_{i}-y_{j}\right) \geq 0$, for any $i, j \in\{1, \ldots, n\}$.

Recall that OWA operators are also averaging, or equivalently, idempotent aggregation functions [9], which means $A(x, \ldots, x)=x$, for any $x \in[0,1]$. Also, OWA operators are positively homogeneous and piece wise linear [9]. However, OWA operators are generally not associative or decomposable [6].

As shown in [8], OWA operators can be seen as Choquet integrals [3] with respect to symmetric capacities too. It should be noted that dealing with a finite universe $X=\{1, \ldots, n\}$, functions $f: X \rightarrow$ $[0,1]$ can be identified with vectors $\mathbf{x} \in[0,1]^{n}, x_{i}=f(i), i=1, \ldots, n$.

Definition 1.1. [9] A capacity (fuzzy measure) $m: \mathcal{P}(X) \rightarrow[0,1]$ is a monotone set function which satisfies in two boundary conditions, $m(\varnothing)=0$ and $m(X)=1$.

Hereafter, we may assume $X=\{1, \ldots, n\}$, unless stated otherwise.

Definition 1.2. [9] For a given vector $\mathbf{x} \in[0,1]^{n}$ and capacity $m$ on $X$, the corresponding Choquet integral is given by

$$
\mathrm{Ch}_{m}(\mathbf{x})=\sum_{i=1}^{n} x_{\sigma(i)}\left(m\left(E_{\sigma, i}\right)-m\left(E_{\sigma, i-1}\right)\right)
$$

where $\sigma:\{1, \ldots, n\} \rightarrow\{1, \ldots, n\}$ is a permutation such that $x_{\sigma(1)} \geq \cdots \geq x_{\sigma(n)}, E_{\sigma, 0}=\emptyset$, and for $i=$ $1, \ldots, n, E_{\sigma, i}=\{\sigma(1), \ldots, \sigma(i)\}$.

It may happen that the permutation $\sigma$ is not unique. This fact does not harm the correctness of (2). In fact, (1) and (2) coincide for each $\mathbf{x} \in[0,1]^{n}$ if and only if $m\left(E_{\sigma, i}\right)$ does not depend on the considered permutation $\sigma$ [8]. This means that only cardinality of $E_{\sigma, i}$ matters, i.e., $m(E)=$ $m(\sigma(E))$, for any $E \in \mathcal{P}(X)$ and permutation $\sigma, \sigma(E)=\{\sigma(i) \mid i \in E\}$. Such capacities are called symmetric. Now, it is enough to put $w_{i}=m\left(E_{\sigma, i}\right)-m\left(E_{\sigma, i-1}\right)$ to see that

$$
\mathrm{OWA}_{\mathbf{w}}=\mathrm{Ch}_{m} \text {. }
$$

Conversely, for any normed weighting vector $\mathbf{w}$, it is enough to define a symmetric capacity $m: \mathcal{P}(X) \rightarrow[0,1]$ by

$$
m(E)=\sum_{i=1}^{\operatorname{card}(E)} w_{i}
$$

to see the representation (3).

Any proper generalization of OWA operators should violate some of the mentioned properties of the OWA operators. In the sequel, we restate some of the generalizations. 


\section{Some Generalization}

A simple but important generalization of OWA operators has been introduced in [20].

Definition 2.1. [20] A mapping $M_{\mathrm{w}, \lambda}:[0,1]^{n} \rightarrow[0,1]$ is called a generalized ordered weighted aggregation (GOWA) operator of dimension $n$ if

$$
M_{\mathbf{w}, \lambda}\left(x_{1}, \ldots, x_{n}\right)=\left(\sum_{i=1}^{n} w_{i} x_{\sigma(i)}^{\lambda}\right)^{\frac{1}{\lambda}}
$$

where $\lambda$ is a parameter such that $\lambda \in]-\infty, 0[\cup] 0,+\infty\left[\right.$. For $\lambda=0, M_{\mathbf{w}, 0}(\boldsymbol{x})=\prod_{i=1}^{n} x_{\sigma(i)}^{w_{i}}$ (the so called power-root operator).

Two special cases are of great significance. First is the case when $\lambda=1$, which gives

$$
M_{\mathbf{w}, 1}\left(x_{1}, \ldots, x_{n}\right)=\sum_{i=1}^{n} w_{i} x_{\sigma(i)}
$$

the standard OWA operator. The other important special case is when $w_{j}=\frac{1}{n}$. In this case, we have

$$
M_{\mathbf{w}, \lambda}\left(x_{1}, \ldots, x_{n}\right)=\left(\sum_{i=1}^{n} \frac{1}{n} x_{\sigma(i)}^{\lambda}\right)^{\frac{1}{\lambda}}
$$

which is the generalized arithmetic mean operator. Note that GOWA operators are commutative, monotone, idempotent and, as a result, averaging operators [20].

Recently, a new kind of OWA generalization based on decomposition integrals has been introduced [13]. This kind of OWA operator is based on the representation of OWA operators as Choquet integrals with respect to symmetric capacities. In the next section, we review them.

\section{1. $\mathcal{H}$-OWA Operates}

Any non-empty set of non-empty subsets of $X$ is called a collection. Any nonempty set $\mathcal{H}$ of collections is called a decomposition system.

Definition 2.2. [5] Let a decomposition system $\mathcal{H}$ be fixed. For a capacity $m$ on $X$, the corresponding $\mathcal{H}$-decomposition integral $I_{\mathcal{H}, m}$ is given by

$$
I_{\mathcal{H}, m}(\mathbf{x})=\max \left\{\sum_{i \in J} a_{i} \cdot m\left(A_{i}\right) \mid\left(A_{i}\right)_{i \in J} \in \mathcal{H}, a_{i} \geq 0, \text { for each } i \in J, \quad \sum_{i \in J} a_{i} \cdot 1_{A_{i}} \leq \mathbf{x}\right\}
$$

Alternatively, we can write 


$$
I_{\mathcal{H}, m}(\mathbf{x})=\max _{\zeta \in \mathcal{H}}\left\{\sum_{A_{i} \in \zeta} a_{i} . m\left(A_{i}\right) \mid a_{i} \geq 0, \text { for each } i \in J, \quad \sum_{A_{i} \in \zeta} a_{i} .1_{A_{i}} \leq \mathbf{x}\right\}=\max _{\zeta \in \mathcal{H}} I_{\zeta, m} .
$$

For any collection $\zeta$, the functional $I_{\zeta, m}$ is positively homogeneous, monotone and piece-wise linear, and thus also the functional $I_{\mathcal{H}, m}$ has these properties. Obviously, it is symmetric whenever the capacity $m$ is symmetric. In general, it need not be idempotent and neither an aggregation function. However, due to the positive homogeneity, the mapping

$$
A_{\mathcal{H}, m}=\frac{I_{\mathcal{H}, m}}{I_{\mathcal{H}, m}(\mathbf{1})}:[0,1]^{n} \rightarrow[0,1]
$$

is an idempotent aggregation function whenever $I_{\mathcal{H}, m}(\mathbf{1})>0$.

Recall that due to [12], $I_{\mathcal{H}, m}(\mathbf{1})=1$, for each capacity $m$, whenever the decomposition system $\mathcal{H}$ is complete (i.e., each non-empty subset $E$ of $X$ is contained in at least one collection from $\mathcal{H}$ ) and any of its collections is formed by logically independent subsets of $X$ (i.e., their intersection is nonempty). Now, we introduce some decomposition systems and related decomposition integrals.

Example 2.1. [13] Let $X=\{1, \ldots, n\}$.

1. Let $\mathcal{H}^{(i)}=\{\mathcal{B} \mid \mathcal{B}$ is a chain in $X$ of length $i\}, \quad i \in\{1, \ldots, n\}$. As shown in [12], these decomposition systems yield the only kind of decomposition integrals which are also universal integrals in the sense of Klement et al. [10]. Note that $I_{\mathcal{H}^{(1)}, m}$ is the Shilkret integral [19], while $I_{\mathcal{H}}{ }^{(n)}, m=\mathrm{Ch}_{m}$ is the Choquet integral [3]. Note that $I_{\mathcal{H}^{(i)}, m}$ is an aggregation function for each capacity $m$ and $i \in\{1, \ldots, n\}$.

2. For $\emptyset \neq A \subseteq X$, let $\mathcal{H}_{A}=\{\{A\}\}$. Then,

$$
I_{\mathcal{H}_{A}, m}(\mathbf{x})=\min \left\{x_{i} \mid i \in A\right\} \cdot m(A) .
$$

and $I_{\mathcal{H}_{A}, m}$ is an aggregation function only if $m(A)=1$.

Example 2.2. Let $X=\{1, \ldots, n\}$. We can introduce collections $G_{i}=\{X\} \cup\{A \mid A \subseteq X$, $\operatorname{card}(A)=i\}$, for $i=0, \ldots, n-1$. Now, we can form the union of these collections which are decomposition systems, $\mathcal{H}_{i}=G_{1} \cup \ldots \cup G_{i}$. Then, $\mathcal{H}_{n-1}=\{A \mid A \subseteq X\}=\mathcal{P}(X)$. Note that for $\mathcal{H}_{i}$ we get maximum of operators, given by (4), corresponding to $G_{1}, \ldots, G_{i}$.

The symmetry of a considered capacity $m$ does not imply the symmetry of decomposition integrals; for a counter example, see [13]. In fact, it is also related to decomposition systems.

Definition 2.3. [13] Let $\sigma: X \rightarrow X$ be a permutation on $X$. For any non-empty $E \subseteq X$, denote $E_{\sigma}=$ $\{\sigma(i) \mid i \in E\}$. For any collection $\mathcal{B}=\left\{E_{1}, \ldots, E_{k}\right\}$, denote $\mathcal{B}_{\sigma}=\left\{\left(E_{1}\right)_{\sigma}, \ldots,\left(E_{k}\right)_{\sigma}\right\}$. Similarly, for any decomposition system $\mathcal{H}$, denote $\mathcal{H}_{\sigma}=\left\{\mathcal{B}_{\sigma} \mid \mathcal{B} \in \mathcal{H}\right\}$. A decomposition system $\mathcal{H}$ is called symmetric if and only if $\mathcal{H}=\mathcal{H}_{\sigma}$ for any permutation $\sigma$ on $X$. 
As an example, decomposition systems $\mathcal{H}^{(i)}$, for all $i=1, \ldots, n$, are symmetric, but it is not the case for systems $\mathcal{H}_{A}$ for $A \subseteq X$.

Definition 2.4. [13] Let $\mathcal{H}$ be a decomposition system on $X$ such that $X \in \mathcal{B}$ for some $\mathcal{B} \in \mathcal{H}$. Then, $\mathcal{H}$ is called a saturated decomposition system.

Observe that the decomposition systems $\mathcal{H}^{(i)}$ are saturated for all $i=1, \ldots, n$, but not the systems $\mathcal{H}_{A}$ for $A \subseteq X$.

Definition 2.5. [13] Let $\mathcal{H}$ be a symmetric saturated decomposition system on $X$, and let $m$ be a symmetric capacity on $X$. Then, the functional $\mathcal{H}-\mathrm{OWA}_{\mathbf{w}}:[0,1]^{n} \rightarrow[0,1]$, given by

$$
\mathcal{H}-\mathrm{OWA}_{\mathbf{w}}(\mathbf{x})=\frac{I_{\mathcal{H}, m}(\mathbf{x})}{I_{\mathcal{H}, m}(\mathbf{1})}
$$

is called a decomposition OWA operator. Here, $\mathbf{w}=\left(w_{1}, \ldots, w_{n}\right)$ with $w_{1}=m(\{1\}), w_{2}=$ $m(\{1,2\})-m(\{1\}), \ldots, w_{n}=m(\{1, \ldots, n\})-m(\{1, \ldots, n-1\})$, i.e.,

$$
m(E)=\sum_{i=1}^{\operatorname{card}(E)} w_{i}, E \in \mathcal{P}(X) .
$$

According to Example 2.1, $\mathcal{H}^{(n)}$ generates the Choquet integral. So, $\mathcal{H}^{(n)}-\mathrm{OWA}_{\mathbf{w}}=\mathrm{OWA}_{\boldsymbol{w}}$ is the standard OWA operator [13].

Example 2.3. [13] Decomposition systems $\mathcal{H}^{(1)}$ and $\mathcal{H}^{(2)}$ are symmetric saturated and their corresponding $\mathcal{H}-\mathrm{OWA}_{\mathbf{w}}$ operators are

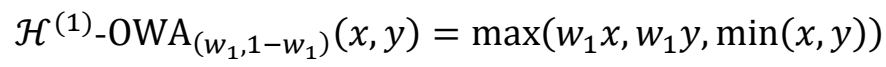

and

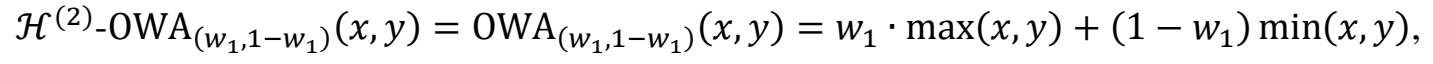

respectively.

This class of aggregation functions has the properties of symmetry, positive homogeneity, idempotency, piecewise linearity, and monotonicity in weights. $\mathcal{H}$-based generalization of OWA operators consist of well-known aggregation functions such as standard OWA operators in special case, arithmetic mean. Also, by this generalization, several new aggregation functions can be obtained [13].

\section{2. $\quad \mathrm{OWA}_{\mathrm{w}}^{(i)}$ Operator}

Definition 2.6. [14] Let $m: \mathcal{P}(X) \rightarrow[0,1]$ be a capacity and let $i \in\{1,2, \ldots, 2 n-1\}$. A productbased integral $I_{m}^{(i)}:[0,1]^{n} \rightarrow[0,1]$ is given by 


$$
I_{m}^{(i)}(\mathbf{x})=\max \left\{\sum_{j=1}^{n} x_{\sigma\left(k_{j}\right)}\left(m\left(E_{\sigma, k_{j}}\right)-m\left(E_{\sigma, k_{j-1}}\right)\right) \mid 1 \leq k_{1}<\cdots<k_{i}=n\right\},
$$

when $i \in\{1, \ldots, n\}$ with convention $k_{0}=0$, and is given by

$$
I_{m}^{(i)}(\mathbf{x})=\min \left\{\sum_{j=1}^{2 n-i} x_{\sigma\left(k_{j}\right)} \cdot\left(m\left(E_{\sigma, k_{j+1}-1}\right)-m\left(E_{\sigma, k_{j-1}}\right)\right) \mid 1 \leq k_{1}<\cdots<k_{2 n-i} \leq n\right\},
$$

when $i \in\{n, \ldots, 2 n-1\}$ with convention

$$
k_{2 n-i+1}=\left\{\begin{array}{lr}
\min \left\{r \mid x_{\sigma(r)}=0\right\}, & x_{\sigma(n)}=0 \\
n+1, & \text { otherwise. }
\end{array}\right.
$$

Observe that if $i=n$, then from (5) or (6), we have $I_{m}^{(n)}=\mathrm{Ch}_{m}$. Also,

$$
I_{m}^{(1)}(\mathbf{x})=\max \left\{x_{\sigma(1)} \cdot m\left(E_{\sigma, 1}\right), \ldots, x_{\sigma(n)} \cdot m\left(E_{\sigma, n}\right)\right\},
$$

which is the Shilkret integral.

Considering normed weighting vector $\mathbf{w} \in[0,1]^{n}$, vector of cumulative weights assigned to $\mathbf{w}$ is vector $\mathbf{v} \in[0,1]^{n}$, where, $v_{1}=w_{1}, v_{2}=w_{1}+w_{2}, \ldots, v_{n}=w_{1}+\cdots+w_{n}$. Now, we can define a generalization of OWA operators based on $I_{m}^{(i)}$.

Definition 2.7. [14] Let $\mathbf{w} \in[0,1]^{n}$ be a normed weighting vector and let $\mathbf{v} \in[0,1]^{n}$ be the related cumulative weighting vector. For $i \in\{1,2, \ldots, 2 n-1\}$, the OWA generalizations $\operatorname{OWA}_{\mathbf{w}}^{(i)}:[0,1]^{n} \rightarrow$ $[0,1]$ are given by

$$
\mathrm{OWA}_{\mathbf{w}}^{(\boldsymbol{i})}(\mathbf{x})=\max \left\{\sum_{j=1}^{n} x_{\left(k_{j}\right)} \cdot\left(v_{k_{j}}-v_{k_{j-1}}\right) \mid 1 \leq k_{1}<\cdots<k_{i} \leq n\right\},
$$

when $i \in\{1, \ldots, n\}$, and is given by

$$
\mathrm{OWA}_{\mathbf{w}}^{(\boldsymbol{i})}(\mathbf{x})=\min \left\{\sum_{j=1}^{2 n-i} x_{\left(k_{j}\right)} \cdot\left(v_{k_{j+1}-1}-v_{k_{j}-1}\right) \mid 1=k_{1}<\cdots<k_{i} \leq n\right\},
$$

when $i \in\{n, \ldots, 2 n-1\}$.

Observe that $\mathrm{OWA}_{\mathbf{w}}^{(n)}=\mathrm{OWA}_{\mathbf{w}}$ is the standard OWA operator and

$$
\mathrm{OWA}_{\mathbf{w}}^{(1)} \leq \mathrm{OWA}_{\mathbf{w}}^{(2)} \leq \cdots \leq \mathrm{OWA}_{\mathbf{w}}^{(2 n-1)}
$$

Note that each $\mathrm{OWA}_{\mathbf{w}}^{(i)}$ is symmetric, positively homogeneous and translation invariant. 
Example 2.4. [14] Consider capacity $m(A)=\frac{|A|}{n}$, constant weighting vector $\mathbf{w}=\left(\frac{1}{n}, \ldots, \frac{1}{n}\right)$, cumulative vector $\mathbf{v}=\left(\frac{1}{n}, \frac{2}{n}, \ldots, \frac{n}{n}\right)$ and input vector $\mathbf{x}=\left(1, \frac{n-2}{n-1}, \ldots, \frac{1}{n-1}, 0\right)$. Then,

$$
\begin{gathered}
\operatorname{OWA}_{\mathbf{w}}^{(\mathbf{1})}(\mathbf{x})=\max \left\{\frac{n-j}{n-1} \cdot \frac{j}{n} \mid j \in\{1, \ldots, n\}\right\}, \\
\operatorname{OWA}_{\mathbf{w}}^{(2)}(\mathbf{x})= \begin{cases}\frac{n}{3(n-1)}, & n=3 k \\
\frac{n+1}{3 n}, & \text { otherwise, }\end{cases} \\
\operatorname{OWA}_{\mathbf{w}}^{(n)}(\mathbf{x})=\frac{1}{2} .
\end{gathered}
$$

\subsection{2-symmetric OWA Operators}

We prepare now a new generalization of OWA operators. In our approach, we generalize the symmetry of a capacity $\mathrm{m}$ into 2 -symmetry; see [15].

Definition 2.8. A capacity $m: \mathcal{P}(X) \rightarrow[0,1]$ is 2 -symmetric whenever there is a set $I \subseteq X$ such that for any $E \subseteq X, m(E)$ depends on $\operatorname{card}(E \cap I)$ and $\operatorname{card}(E \backslash I)$ only.

Obviously, if $I=\varnothing$ or $I=X$, then the related 2-symmetric capacity $m$ is a standard symmetric capacity. Denote $\operatorname{card}(I)=k$. Clearly, $k \in\{0,1, \ldots, n\}$ and for $k \in\{0,1, \ldots, n-1\}$ (i.e., $I$ is a proper subset of $X), m$ can expressed by means of a monotone function as

$$
m(E)=h((\operatorname{card}(E \cap I), \operatorname{card}(E \backslash I))),
$$

where $h: D_{k} \rightarrow[0,1], D_{k}=\{(i, j) \mid i \in\{0, \ldots, k\}, j \in\{0, \ldots, n-k\}\}, h((0,0))=0$ and $h((k, n-$ $k))=1$.

Now, we present the definition of 2-symmetric OWA operator.

Definition 2.9. Let $m: \mathcal{P}(X) \rightarrow[0,1]$ be a 2 -symmetric capacity. Then, the related Choquet integral $\mathrm{Ch}_{m}:[0,1]^{n} \rightarrow[0,1]$, given by

$$
\mathrm{Ch}_{m}(\mathbf{x})=\sum_{i=1}^{n} x_{\sigma(i)}\left(h\left(\left(i^{\prime}, i-i^{\prime}\right)\right)-h\left(\left((i-1)^{\prime},(i-1)-(i-1)^{\prime}\right)\right)\right) \text {, }
$$

is called a 2-symmetric OWA operator.

Also, 2-symmetric OWA operators can be obtained in a different way. Take a subset $I$ of $\{1, \ldots, n\}$, $I=\left\{i_{1}, \ldots, i_{k}\right\}$, and its complement $I^{c}=\left\{j_{1}, \ldots, j_{n-k}\right\}$. We have the following definition. 
Definition 2.10. An aggregation function $A:[0,1]^{n} \rightarrow[0,1]$ is called 2-symmetric whenever for each $\mathbf{x} \in[0,1]^{n}$, we have $A(\mathbf{x})=A\left(\mathbf{x}_{\sigma}\right)$ for any of four permutations $\sigma \in\{\alpha, \beta, \gamma, \delta\}$ such that

$$
\begin{aligned}
& \alpha\left(i_{1}\right)=i_{2}, \alpha\left(i_{2}\right)=i_{3}, \ldots, \alpha\left(i_{k-1}\right)=i_{k}, \alpha\left(i_{k}\right)=i_{1}, \\
& \beta\left(i_{1}\right)=i_{2}, \beta\left(i_{2}\right)=i_{1}, \beta\left(i_{3}\right)=i_{3}, \ldots, \beta\left(i_{k}\right)=i_{k}, \\
& \gamma\left(j_{1}\right)=j_{2}, \gamma\left(j_{2}\right)=j_{3}, \ldots, \gamma\left(j_{n-k-1}\right)=j_{n-k}, \gamma\left(j_{n-k}\right)=j_{1}, \\
& \delta\left(j_{1}\right)=j_{2}, \delta\left(j_{2}\right)=j_{1}, \delta\left(j_{3}\right)=j_{3}, \ldots, \delta\left(j_{n-k}\right)=j_{n-k} .
\end{aligned}
$$

Due to [17], this means that $A(\mathbf{x})=A\left(\mathbf{x}_{\sigma}\right)$ for any permutation $\sigma$ such that $\sigma(i) \in I$ for any $i \in I$ and $\sigma(j) \in I^{c}$ for any $j \in I^{c}$.

Now, we can define 2-symmetric OWA operator 2-OWA as a 2-symmetric comonotone additive aggregation function. Due to comonotone additivity, clearly it is a Choquet integral with respect to a capacity $m, m(E)=2$-OWA $\left(\mathbf{1}_{E}\right)$, and this capacity is 2-symmetric in the sense of [15]. As also the opposite claim is true, we have the following theorem.

Theorem 2.1. An aggregation function is a 2-OWA operator if and only if it is Choquet integral with respect to some 2-symmetric capacity $m$.

Note that one can define $p$-symmetric aggregation functions in which $p \geq 2$.

\section{Conclusion}

The concept of OWA operators and some of their generalizations were reviewed. Also, the definition of 2-symmetric OWA operators was introduced.

\section{Acknowledgement}

The second author acknowledges support from APVV-14-0013 and VEGA 1/0420/15 research grants.

\section{References}

[1] Ahn, B.S. (2009), Some remarks on the LSOWA approach for obtaining OWA operator weights, International Journal of Intelligent Systems, 24(12), 1265-1279.

[2] Beliakov, G. Pradera A., and Calvo, T. (2007), Aggregation Functions: A Guide for Practitioners, Springer, Berlin.

[3] Choquet, G. (1953), Theory of capacities, Annales de l'Institut Fourier, 5, 131-295

[4] Emrouznejad, A., and Marra, M. (2014), Ordered weighted averaging operators, 19882014: a citation-based literature survey, International Journal of Intelligent Systems, 29, 994-1014.

[5] Even, Y., and Lehrer, E. (2014), Decomposition-integral: unifying Choquet and the concave integrals, Economic Theory, 56(1), 1-26.

[6] Fodor, J. Marichal, J.L., and Roubens, M. (1995), Characterization of the ordered weighted averaging operators, IEEE Transactions on Fuzzy Systems, 3(2), 236-240.

[7] Fuller, R. (1996), OWA operators in decision making, In: C. Carlsson (Ed.), Exploring the Limits of Support Systems, TUCS General Publications No. 3, Turku Center for Computer Science, Abo, 85-104. 
[8] Grabisch, M. (1995), Fuzzy integral in multicriteria decision making, Fuzzy Sets and Systems, 69(3), 279-298.

[9] Grabisch, M. Marichal, J.L. Mesiar, R., and Pap, E. (2009), Aggregation functions (encyklopedia of mathematics and its applications), Cambridge University Press.

[10] Klement, E.P. Mesiar, R., and Pap, E. (2010), A universal integral as common frame for Choquet and Sugeno integral, IEEE Transactions on Fuzzy Systems, 18, 178-187.

[11] Merigo, J.M. (2012), OWA operators in the weighted average and their application in decision making, Control and Cybernetics, 41(3), 605-643.

[12] Mesiar, R., and Stupňanová, A. (2013), Decomposition integrals, International Journal of Approximate Reasoning, 54, 1252-1259.

[13] Mesiar, R., and Stupňanová, A. (2016), Decomposition integral based generalizations of OWA operators, Information Processing and Management of Uncertainty in KnowledgeBased Systems, Volume 610 of the series Communications in Computer and Information Science, 3-10.

[14] Mesiar, R., and Stupňanová, A. Yager, R.R. (2015), Generalizations of OWA operators, IEEE Transactions on Fuzzy Systems, 23(6), 2154-2162.

[15] Miranda, P. Grabisch, M., and Gil, P. (2002), p-symmetric fuzzy measures, International Journal of Uncertainty, Fuzziness and Knowledge-Based Systems, 10, 105-123.

[16] Reimann, O. Schumacher, C., and Vetschera, R. (2017), How well does the OWA operator represent real preferences?, European Journal of Operational Research, 258(3), 993-1003.

[17] Rotman, J.J. (2005), An Introduction to the Theory of Groups, 4th Edition, Springer, New York.

[18] Schmeidler, D. (1989), Subjective probability and expected utility without additivity, Econometrica, 57, 571-587.

[19] Shilkret, N. (1971), Maxitive measure and integration, Indagationes Mathematicae, 33, 109-116.

[20] Yager, R.R. (2004), Generalized OWA aggregation operators, Fuzzy Optimization and Decision Making, 3, 93-107.

[21] Yager, R.R. (1988), On ordered weighted averaging aggregation operators in multicriteria decision making, IEEE Transactions on Systems, Man. and Cybernetics, 18, 183-190.

[22] Yager, R.R. (2009), Weighted maximum entropy OWA aggregation with application to decision making under risk, IEEE Transactions on Systems, Man. and Cybernetics, 39, 555564. 